\title{
Caloric method for the energetic evaluation of decentralised domestic ventilation devices
}

\author{
Christian Friebe ${ }^{1, *}$, Andreas Hantsch $^{1}$, Sabine Döge ${ }^{1}$ and Ralph Krause ${ }^{1}$ \\ ${ }^{1}$ Institut für Luft- und Kältetechnik gGmbH, Hauptbereich Klimatechnik, 01309 Dresden, Germany
}

\begin{abstract}
In the context of this article, a caloric method for measuring the performance of alternating home ventilation devices (push-pull units) is presented. The simple and robust method provides both reliable and reproducible characteristic values. Based on this method, a test bench was developed and built. With this test bench the characteristic values (volume flow, heat recovery rate, temperature change rate) of series products can be measured. The aim of the investigation is the determination and evaluation of the possible parameters influencing the measurement. For this purpose, the parameters are illustrated on the basis of measured values from a defined test standard, consisting of a fan and electrical heating in a housing (so-called golden sample), as well as a pair of push-pull devices. As a result, suggestions for improvements to the previous procedure as well as approaches for further development are shown.
\end{abstract}

\section{Motivation}

\subsection{Working principle}

Due to the increasing demands on energy efficiency in the construction sector, tightness and thermal insulation are constantly increasing. Highly efficient ventilation solutions are therefore required, especially in the field of building refurbishment. Alternating home ventilation devices (push-pull units) have advantages due to their working principle and easy installation procedure at this field.

Consisting on one fan and a sensitive heat accumulator, the direction of the air mass flow is changed by reversing the fans direction of rotation over time $\tau$. A typical behaviour is represented in Fig. 1. Where $B$ is the mode of operation ( $B=1$ air from ambient into the room, $B=-1$ from room to ambient), $\dot{m}$ as mass flow and $T$ as temperature with ETH as extraction air, EHH as exhaust air, SUP as supply air and ODA as outdoor air. For balancing reasons, two units are used mostly with one unit working in mode $B=1$ and the other in mode $B=-1$ at the same time. The air temperature at the indoor side of the unit is denoted by $i$ and at the outdoor side by $a$.

Assuming winter season, in mode $B=-1$ the heat accumulator is heated up by the warm indoor air. The temperature at the outdoor side of the unit is decreased by this energy transfer. The energy stored within the accumulator is then transferred to the outdoor air at $B=1$.

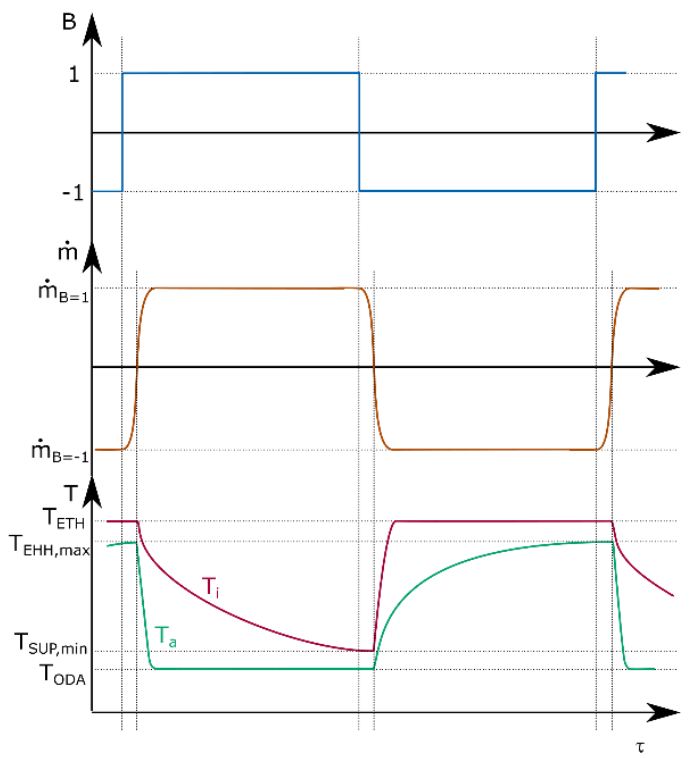

Fig. 1. Schematically representation of a typical push-pull unit behaviour.

\subsection{Efficiency definitions}

The efficiency of push-pull ventilation units is defined by the ratio of heating capacity with heat recovery and without heat recovery. The calculation is defined with different formulations within different standards (VDI 3803-5 [1], DIN EN 308 [2], DIN 4719 [3] and DIN EN 13141-8 [4]).

\footnotetext{
*Corresponding author: christian.friebe $@$,ilkdresden.de
} 


\section{Measurement principle}

\subsection{Direct measurement method}

EN 13141-8 [4] describes a measurement method utilizing temperature probes at the inlet and outlet of one unit. The efficiency $\eta_{t \text {,SuP }}$ is calculated according to eq. (1) based on these measured temperatures $T$ and mass flows $\dot{m}$.

$$
\eta_{t, \mathrm{SUP}}=\frac{T_{\mathrm{SUP}}-T_{\mathrm{ODA}}}{T_{\mathrm{ETH}}-T_{\mathrm{ODA}}} \frac{\dot{m}_{\mathrm{m}, \mathrm{SUP}}}{\dot{m}_{\mathrm{m}, \mathrm{ETH}}}
$$

\subsection{Mixed air measurement method}

Another attempt utilizing a temperature measurement at mixed air at two different modes (constant flow mode, indicated by s, and alternating flow mode) is described by Özbiyik [5]. Eq. (2) presents the description of the efficiency obtained by this method. Here, 1 denotes inlet conditions and 2 outlet conditions.

In case of same mass flows eq. (2) simplifies to eq. (3). This equation is normally used, but some effects are neglected.

$$
\begin{aligned}
& \eta_{t, \mathrm{SUP}} \\
& =\frac{1}{t_{\mathrm{ETH}, 1}-\frac{\dot{m}_{\mathrm{ETH}, 2, s} t_{\mathrm{ETH}, 2, s}}{\dot{m}_{\mathrm{SUP}, s}}-\left(1-\frac{\dot{m}_{\mathrm{ETH}, 1, s}}{\dot{m}_{\mathrm{SUP}, s}}\right) t_{\mathrm{ETH}, 1, \mathrm{~s}}} \\
& \left(\frac{\dot{m}_{\mathrm{ETH}, 2} t_{\mathrm{ETH}, 2}}{\dot{m}_{\mathrm{SUP}}}-\frac{\dot{m}_{\mathrm{ETH}, 2, s} t_{\mathrm{ETH}, 2, s}}{\dot{m}_{\mathrm{SUP}, s}}\right. \\
& -\left(1-\frac{\dot{m}_{\mathrm{ETH}, 1, s}}{\dot{m}_{\mathrm{SUP}, s}}\right) t_{\mathrm{ETH}, 1, s} \\
& \left.+\left(1-\frac{\dot{m}_{\mathrm{ETH}, 1}}{\dot{m}_{\mathrm{SUP}}}\right) t_{\mathrm{ETH}, 1}\right) \\
& \eta_{t, \mathrm{SUP}}=\frac{t_{\mathrm{ETH}, 2}-t_{\mathrm{ETH}, 2, \mathrm{~s}}}{t_{\mathrm{ETH}, 1}-t_{\mathrm{ETH}, 2, \mathrm{~s}}}
\end{aligned}
$$

\subsection{Caloric measurement principle}

A further attempt utilizes a caloric measurement method. Here, the needed energy to establish constant temperatures in a test chamber is measured directly (Fig. 2).

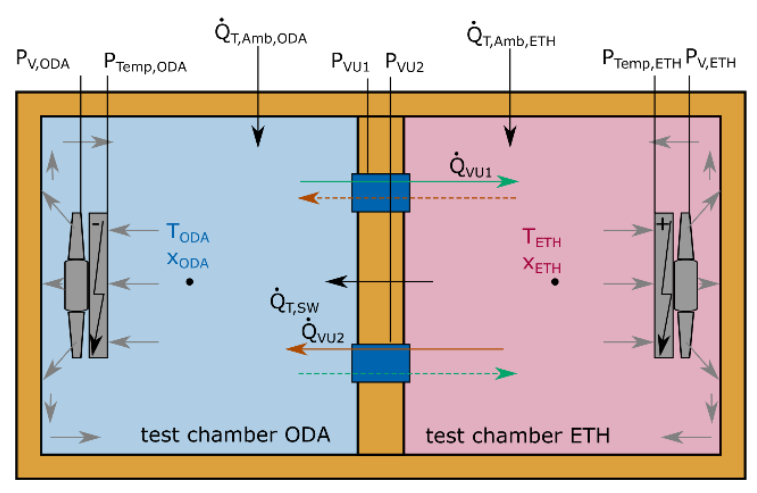

Fig. 2. Schematically representation of the caloric measurement principle.

According to Eq. (4) the efficiency depends upon the relation of the thermal capacity $\dot{Q}_{V U}$ for constant indoor temperature with working ventilation units and a reference thermal capacity $\dot{Q}_{R e f}$.

$$
\eta_{t, S U P}=\frac{\dot{Q}_{V U}}{\dot{Q}_{R e f}}
$$

The reference heat capacity may be defined in two different manners. The first one is using thermal capacity given by the mass flux and the temperature difference between indoor and outdoor conditions and leads to Eq. (5).

$$
\eta_{t, S U P}=\frac{-P_{V, E T H}-P_{T e m p, E T H}-\dot{Q}_{T, E T H}-P_{V U}}{\dot{m}_{\mathrm{m}} c_{p}\left(T_{O D A}-T_{E T H}\right)}
$$

The second definition uses a reference measurement. This reference measurement is performed with ventilation units that operate with a constant, non-alternating mass flow.

$$
\begin{aligned}
& \eta_{t, \text { SUP }} \\
& =\frac{-P_{V, E T H}-P_{T e m p, E T H}-\dot{Q}_{T, E T H}-P_{V U}}{-P_{V, E T H, R e f}-P_{T e m p, E T H, R e f}-\dot{Q}_{T, E T H}-P_{V U, R e f}}
\end{aligned}
$$

\section{$2.4 \quad$ Measurement uncertainties}

For all three attempts to measure the efficiency of pushpull units a measurement uncertainties analysis has been carried out by means of the Gaussian error propagation law [6]. In Eq. (7) $x$ represents a variable of the function $y$ and the uncertainty of the this function $\Delta y$.

$$
y\left(x_{i}\right) \rightarrow \Delta y\left(x_{i}\right)=\sqrt{\sum_{i}\left(\frac{\partial y}{\partial x_{i}} \Delta x_{i}\right)^{2}}
$$

Typical measurement uncertainties are defined in EN 13141-8 [4].

The results obtained by the analysis are provided in Table 1. 
Table 1. Uncertainties of efficiency by different measurement principles.

\begin{tabular}{|c|c|c|c|}
\hline Method & $\begin{array}{l}\text { Equipment } \\
\text { uncertainty }\end{array}$ & Value & $\begin{array}{c}\text { Resulting } \\
\text { uncertainty }\end{array}$ \\
\hline & & $\%$ & $\%$ \\
\hline Direct, eq. (1) & typical & 0,615 & $\pm 0,025$ \\
\hline Direct, eq. (1) & EN 13141-8 & 0,615 & $\pm 0,049$ \\
\hline Mixed air, eq. (3) & typical & 0,758 & $\pm 0,028$ \\
\hline Mixed air, eq. (3) & EN 13141-8 & 0,758 & $\pm 0,041$ \\
\hline Mixed air, eq. (2) & typical & 0,75 & $\pm 0,068$ \\
\hline Caloric, Eq. (5) & typical & 0,655 & $\pm 0,014$ \\
\hline Caloric, Eq. (6) & typical & 0,667 & $\pm 0,0065$ \\
\hline
\end{tabular}

\section{Measurement}

\subsection{Setup}

The company Aereco GmbH (Germany) developed a test rig for the caloric measurement method according to section 2.3, but with on chamber (this test bench is called "BEMdl"). A schematically representation is provided in Fig. 3. This test rig consists of a housing with thermal insulation surrounding a test chamber with two walls, each with a mixing fan and a heater mounted in the center. The walls have a defined space to the housing. At the middle of the chamber a temperature probe is positioned.

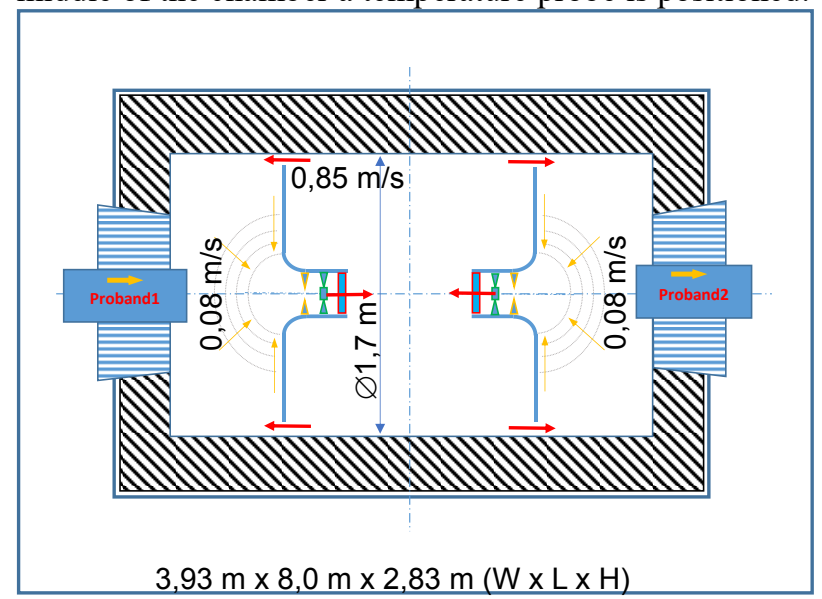

Fig. 3. Working principle of the test rig under investigation.

This test rig is meant for stand-alone measurements at ambient temperature as the cold outdoor temperature and with the indoor temperature $13 \mathrm{~K}$ above the ambient temperature. The test rig was installed within a climate chamber for the purpose of investigating the test rig behaviour upon different conditions (see Fig. 4.)

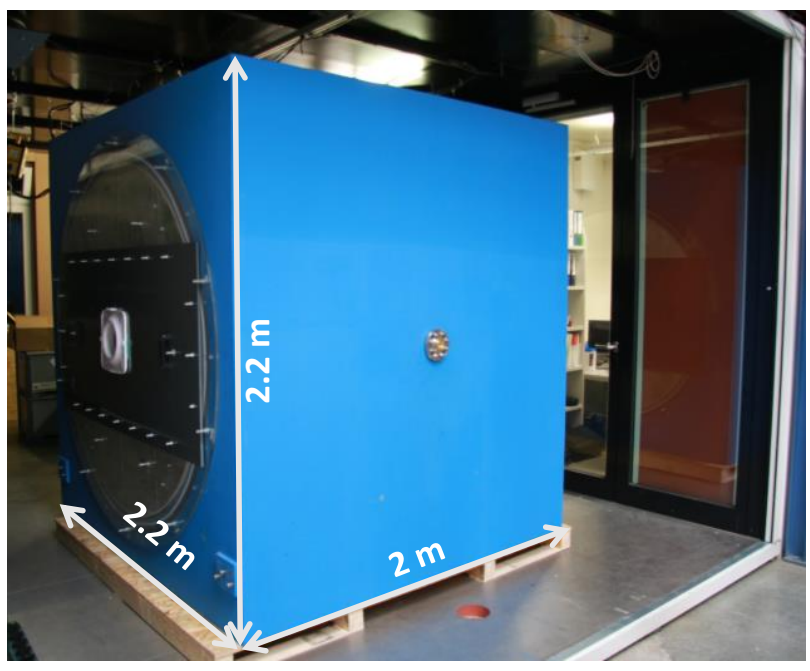

Fig. 4. Test rig within the test chamber.

The test rig is equipped with two temperature probes (indoor and outdoor temperature), one difference pressure gauge (static pressure difference between indoor and outdoor), ambient pressure gauge, mass flow measurement by means of tracer gas measurement, electrical consumption of heaters, mixing fans and ventilation units.

The climate chamber with a size of $3.93 \times 8.00 \times 2.83 \mathrm{~m}^{3}$ is equipped with further sensors. Among these 18 temperature probes (PT100), three difference pressure sensors, one ambient pressure sensor and two sensors for relative humidity are utilized to achieve deeper insights into the measurement principle of the test rig.

\subsection{Verification}

For the purpose of the test rig verification, a so called golden sample was used. This golden sample consist of a fan and an electrical heater and replaces one of the ventilation units. The other ventilation unit was dismounted. Since the electrical power consumption of the fan and the heater is known, the test bench can be validated by the reduction of the heating power required to reach the necessary interior temperature.

\section{3 $\quad$ Test schedule}

Several individual tests were carried out to quantify the influences on the measurement method. Table 2 provides an overview of these tests. Each test was performed with ventilation units in constant flow mode to obtain the reference power consumption and in alternating ventilation mode. Both tests lasted an hour after the conditions stabilized. Different conditions have been investigated. The objective of this work is to investigate the influence of wind pressure, temperature level and unbalanced mass flow. 
Table 2. Test overview

\begin{tabular}{|c|c|c|c|c|}
\hline No. & tier & $\begin{array}{c}\text { Rev. } \\
\text { speed }\end{array}$ & $\begin{array}{c}\text { Temperature } \\
\text { level }\end{array}$ & $\begin{array}{c}\text { Wind } \\
\text { pressure }\end{array}$ \\
\hline $\mathbf{1}$ & 0 & $\mathbf{r p m}$ & ${ }^{\circ} \mathbf{C}$ & $\mathbf{P a}$ \\
\hline $\mathbf{2}$ & 0 & $850 / 650$ & 20 & 0 \\
\hline $\mathbf{3}$ & 1 & $1170 / 1060$ & 7 & 0 \\
\hline $\mathbf{4}$ & 1 & $1170 / 1060$ & 20 & 0 \\
\hline $\mathbf{5}$ & 2 & $1990 / 1840$ & 7 & 0 \\
\hline $\mathbf{6}$ & 2 & $1990 / 1840$ & 7 & 10 \\
\hline $\mathbf{7}$ & 2 & $1990 / 1840$ & 7 & 20 \\
\hline $\mathbf{8}$ & 2 & $1990 / 1840$ & 20 & 0 \\
\hline $\mathbf{9}$ & 2 & $1990 / 1840$ & 20 & 0 \\
\hline & 2 & $1990 / 1840$ & 20 & 0 \\
\hline
\end{tabular}

\section{Results}

\subsection{Definition of efficiency}

Further investigations are carried out using the Eq. (8) to (11). Herein Eq. (8) and Eq. (9) are based upon enthalpy flow and Eq. (10) and (11) are utilizing a correction scheme by implementing the volume flow ratio between the measurement with constant volume flow and alternating volume flow.

$$
\begin{gathered}
\eta_{H R, E S V}=1-\frac{P_{T e m p}+P_{V}-\dot{Q}_{T}+P_{V U}}{\varrho c_{p} \dot{V}_{1}\left(T_{S U P}-T_{O D A}\right)} \\
\eta_{T, E S V}=1-\frac{P_{T e m p}+P_{V}-\dot{Q}_{T}}{\varrho c_{p} \dot{V}_{1}\left(T_{S U P}-T_{O D A}\right)} \\
\eta_{\mathrm{HR}, \mathrm{KV}}=1-\frac{P_{\mathrm{Temp}}+P_{\mathrm{V}}-\dot{Q}_{\mathrm{T}}+P_{\mathrm{VU} 1}}{K\left(P_{\mathrm{Temp}, \mathrm{s}}+P_{\mathrm{V}}-\dot{Q}_{\mathrm{T}}+P_{\mathrm{VU} 1, \mathrm{~s}}\right)} \\
\eta_{\mathrm{T}, \mathrm{KV}}=1-\frac{P_{\mathrm{Temp}}+P_{\mathrm{V}}-\dot{Q}_{\mathrm{T}}}{K\left(P_{\mathrm{Temp}, \mathrm{s}}+P_{\mathrm{V}}-\dot{Q}_{\mathrm{T}}+P_{\mathrm{VU} 1, \mathrm{~s}}\right)}
\end{gathered}
$$

Wherein $K$ is defined as

$$
K=\frac{\dot{V}}{\dot{V}_{\mathrm{s}}} .
$$

\subsection{Influence of ambient temperature level}

The influence of the temperature level (colour coded) is depicted in Fig. 5 depending upon the level of rotational speed (coded as tier level). As one may see, the efficiency is independent from the temperature level. Due to measurement reasons, no measurements have been performed at the tier " 0 ".
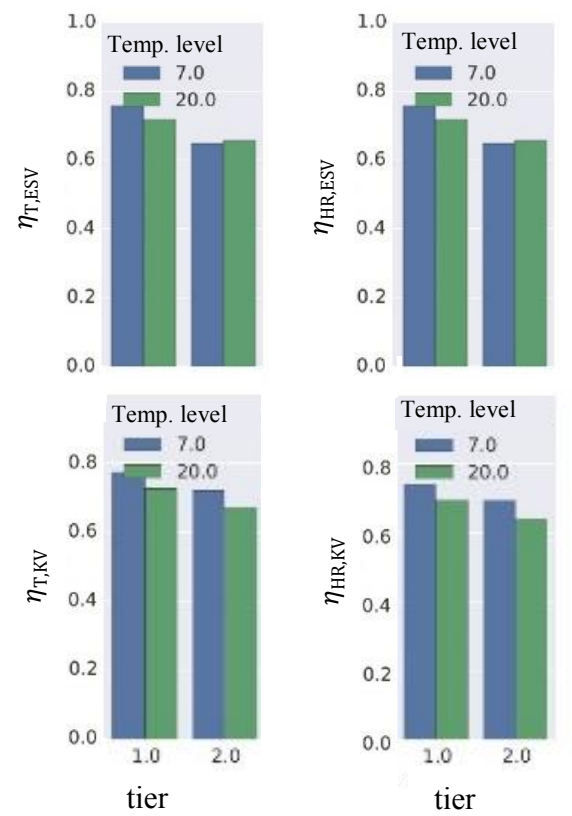

Fig. 5. Influence of ambient temperature.

\subsection{Wind pressure}

The influence of the wind pressure upon the efficiency is depicted in Fig. 6. presenting the influence at a temperature level of $7^{\circ} \mathrm{C}$ as outdoor temperature and $20^{\circ} \mathrm{C}$ indoor temperature. The level of the rotational speed (tier) is color-coded.

The pressure difference between indoor and outdoor conditions has a large influence upon the measured efficiency, especially at low volume flow rates. Increasing wind pressure leads always to lower efficiency ratios. 


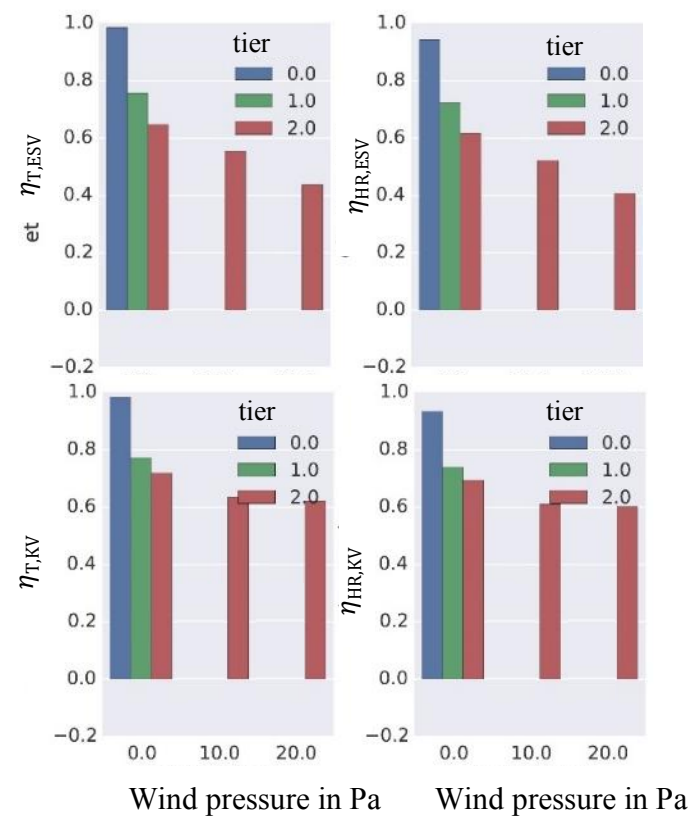

Fig. 6. Influence of wind pressure at temperature level $7 / 20^{\circ} \mathrm{C}$.

\subsection{Unbalanced mass flow rate}

An unbalanced mass flow between both ventilation units leads to a pressure difference between the test bench chamber and the environment. The relationship between pressure difference and mass flow deviation depends upon the characteristic curve of the fan. This relationship is individual for each unit to be measured in the test bench. The value of the pressure difference is suitable as a quality criterion for ventilation units.

\subsection{Golden sample verification}

For all verification measurements the energy provided by the golden sample is $68 \mathrm{Wh}$. The energy reduction measured at the test rig is presented in Table 3. Apart from the first measurement, the measured energy reduction fits very well the golden sample value. According to these results, the uncertainty of the test rig measurement for the power consumption is better than $3.5 \%$. The reasons for the discrepancy of the first values in the first measurement is currently unclear.
Table 3. Measured energy reduction at the test rig by means of golden sample

\begin{tabular}{|c|c|c|}
\hline Temperature level & $\begin{array}{c}\text { Fan control } \\
\text { voltage }\end{array}$ & $\begin{array}{c}\text { Obtained energy } \\
\text { reduction }\end{array}$ \\
\hline${ }^{\circ} \mathbf{C}$ & $\mathbf{V}$ & $\mathbf{W h}$ \\
\hline 7 & 7.5 & 59.7 \\
\hline 7 & 20 & 68.0 \\
\hline 20 & 7.5 & 65.8 \\
\hline 20 & 20 & 69.5 \\
\hline
\end{tabular}

\section{Summary and Discussion}

Whilst all methods provide reliable efficiency ratios and are suitable for the measurement of ventilation units with changing mass flow direction, the method with the lowest uncertainty is the caloric measurement method according to the power propagation law.

The influence of ambient conditions (temperature level) on volume flows below $25 \%$ of the nominal volume flow should be investigated in further projects.

The installation of axial fans causes a high sensitivity of the volume flow and the efficiency in case of pressure differences, e.g. due to wind pressure on façades.

Unbalanced mass flow conditions between supply and exhaust air mass flow causes a pressure difference in the test bench between the inner chamber and the environment. This pressure is to be regarded as a criterion for the quality of balanced mass flows.

Humidity was not in the scope if this work. Therefore, further investigations shall be established to get deeper insight on the dependency upon humidity. Also one aspect for further investigations maybe the dependency of noise emission upon different conditions.

\section{Acknowledgements}

This work has been supported by the Federal Ministry for Economic Affairs and Energy of the Federal Republic of Germany under Contracts no. MF170011 and the company Aereco GmbH, Germany.

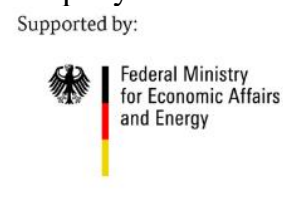
on the basis of a decision
by the German Bundestag 


\section{References}

1. VDI 3803 Blatt 5:2013-04 Air-conditioning, system requirements - Heat recovery systems (VDI Ventilation Code of Practice)

2. DIN EN 308:1997 Heat exchangers - Test procedures for establishing performance of air to air and flue gases heat recovery devices

3. DIN 4719:2009-07: Ventilation and air conditioning - Requirements, performance testing and labelling

4. DIN EN 13141-8:2014: Ventilation for buildings Performance testing of components/products for residential ventilation - Part 8: Performance testing of un-ducted mechanical supply and exhaust ventilation units (including heat recovery) for mechanical ventilation systems intended for a single room

5. T. Özbiyik: Ringversuch zum Vergleich des Prüfverfahrens für dezentrale Lüftungs-geräte mit alternierender Ventilatordrehrichtung zwischen drei Prüfstellen zur energetischen Bewertung : Fraunhofer IRB Verlag, 2017 — ISBN 978-3-8167-9887-3

6. I. N. Bronstein, K. A. Semendjajew, G. Musiol, H. Mühlig: Taschenbuch der Mathematik. 5. ed. Frankfurt am Main : Verlag Harri Deutsch, 2001

7. M. Ben Rabha, M.F. Boujmil, M. Saadoun, B. Bessaïs, Eur. Phys. J. Appl. Phys. (to be published)

8. Luigi T. De Luca, Propulsion physics (EDP Sciences, Les Ulis, 2009)

9. F. De Lillo, F. Cecconi, G. Lacorata, A. Vulpiani, EPL, 84 (2008) 